\title{
A review of the use of high-flow nasal cannula oxygen therapy in hospitalised children at a regional hospital in the Cape Town Metro, South Africa
}

\author{
E Hoffman, MB ChB, DCH; K L Reichmuth, MB ChB, MPH; M L Cooke, MB ChB, FC Paed (SA) \\ Department of Paediatrics and Child Health, Faculty of Health Sciences, University of Cape Town, South Africa
}

Corresponding author: M L Cooke (louise.cooke@uct.ac.za)

Background. High-flow nasal cannula (HFNC) oxygen is a non-invasive alternative to nasal continuous positive airway pressure (CPAP) therapy for infants and children requiring respiratory support. There is a paucity of data to support its use in children, with no published data from sub-Saharan Africa.

Objectives. To describe the outcomes of and adverse events related to HFNC in the first year of its use in a level 2 (L2) general paediatric ward, and to compare these outcomes with those of a historical cohort when this intervention was unavailable.

Methods. This retrospective descriptive study included children aged $<13$ years who received HFNC in the first 12 months after its introduction (HFNC-availability group, $n=66$ ). Demographic data, clinical characteristics and outcomes (death, treatment failure, length of HFNC and HFNC-related adverse events) were assessed. A comparative description of children who required transfer to level 3 (L3) for any form of respiratory support (other than the available standard low-flow oxygen) during the 12-month period prior to HFNC availability (pre-HFNC group, $n=54$ ) was made. All analyses were performed in the paediatric wards, New Somerset Hospital, Cape Town, South Africa. Outcomes were compared using standard descriptive and comparative statistics.

Results. The median age of the cohort was 5 months (interquartile range (IQR) 1.9 - 14.6). Sixteen children (13.3\%) were malnourished, 10 (8.3\%) were HIV-infected, and 30 (25.0\%) had been born prematurely. The most common diagnoses were pneumonia, bronchiolitis and asthma. Asthma, anaemia and cardiac abnormalities were the most prevalent underlying comorbidities. Two children died in each group. All 54 children in the pre-HFNC group were transferred to L3; 38 (70.4\%) needed CPAP or invasive ventilation. In the HFNC-availability period, 85 children were assessed as needing more than standard low-flow oxygen therapy: of the 19 immediately transferred to L3, $17(89.4 \%)$ received CPAP or invasive ventilation; of the 66 who received HFNC at L2, 16 (24.2\%) subsequently required transfer to L3 for CPAP or invasive ventilation. The median duration of HFNC was 46.3 hours (IQR 19.5 - 93.5) overall, and it was 12 hours (IQR 4 - 28) and 58.5 hours (IQR 39.5 - 106) for those who failed or were successfully managed on HFNC, respectively. No HFNC-related serious adverse events were recorded.

Conclusions. HFNC is a safe, effective, feasible option for non-invasive ventilation of children with respiratory illnesses in a resource-limited L2 setting. A greater proportion of children with lower respiratory tract infections in the HFNC-availability group than in the pre-HFNC group required support, but the intervention reduced the bed pressure on L3. Improved ways to identify HFNC failures would be beneficial.

S Afr Med J 2019;109(4):272-277. DOI:10.7196/SAMJ.2019.v109i4.13145

Acute severe lower respiratory tract infections (LRTIs) continue to be a major cause of mortality $(13 \%)$ and morbidity globally ${ }^{[1,2]}$ and in South Africa (SA). ${ }^{[1]}$ Reid et al. ${ }^{[3]}$ showed that pneumonia was the main cause of death $(25 \%)$ in children aged $<5$ years in the Metro West health district of the Western Cape Province of SA. ${ }^{[3]}$ In many middle- to low-income countries with resource limitations, the number of child deaths resulting from severe pneumonia (related to hypoxaemia and respiratory failure) remains high despite implementation of international and local guidelines. ${ }^{[4]}$ Additional respiratory support, important in the care of critically ill children, is often unavailable or is perceived as being neither feasible nor safe in resource-limited settings. ${ }^{[4,5]}$ The use of non-invasive ventilation for respiratory support, including nasal continuous positive airway pressure (CPAP) and high-flow nasal cannula (HFNC) therapy, in and outside the paediatric intensive care unit (PICU) is increasing. ${ }^{[5,6]}$ There is limited evidence to support the use of HFNC in children outside the PICU setting and in conditions other than bronchiolitis. ${ }^{[7]}$

The probable mechanisms of action of HFNC include heating and humidification of inspired air, nasopharyngeal carbon dioxide wash- out, reduction in upper airway resistance, and provision of positive distending pressure. ${ }^{[8-12]}$ The current paucity of data on the variable positive distending pressure provided by HFNC raises clinical safety concerns, ${ }^{[7]}$ and many modern HFNC devices therefore now have pressure-limiting valves. However, more robust physiological studies are needed. ${ }^{[7]}$

A pioneering, contextually relevant study from Bangladesh showed that outcomes in terms of 'treatment failure' (defined as two or three of the following characteristics: severe hypoxia, signs of severe clinical distress, or respiratory acidosis on blood gas analysis) were no worse in patients with severe pneumonia who received HFNC than in those who received bubble CPAP, but mortality rates were higher in the low-flow nasal cannula (LFNC) and HFNC groups, indicating a potential risk of harm associated with these modalities. ${ }^{[13]}$ Various observational studies have shown that HFNC reduces respiratory rates and the effort of breathing in infants with bronchiolitis, has very few reported complications, and is well tolerated. ${ }^{[7]}$

Commercial HFNC devices and circuits are expensive. Guaranteeing that a clinically effective and cost-effective form of 
respiratory support is delivered in a safe environment and that children requiring escalation of respiratory support are identified early is crucial to the appropriate utilisation of a new intervention, especially in a resource-limited environment. Consequently, more research is needed to document efficacy and safety in various clinical scenarios.

\section{Objectives}

The donation in 2015 of two commercial HFNC devices (Fisher and Paykel Airvo 2) to the general paediatric ward at New Somerset Hospital (NSH), a level 2 (L2) hospital in the Metro West subdistrict of Cape Town, SA, afforded the pragmatic opportunity to review this new intervention. Prior to the availability of on-site HFNC, all children who may have required additional respiratory support (HFNC, CPAP or intermittent positive-pressure ventilation (IPPV)) were transferred by ambulance to a level 3 (L3) children's hospital that has dedicated high-care and PICU facilities. The main objective of this study was to document the first year of experience using HFNC by describing the demographic and clinical characteristics of children who were initiated on HFNC oxygen therapy on site, evaluating their response to the intervention and assessing its safety. Our secondary objective was to compare the outcomes in this group with those in a group transferred to L3 for respiratory support during the year before HFNC was available on site.

\section{Methods}

\section{Study design and participants}

This was a retrospective descriptive study of children aged $<13$ years hospitalised with a respiratory condition for which they were managed with HFNC oxygen therapy in the general paediatric ward of NSH during the first 12 months of its availability (1 August 2015 - 31 July 2016). Comparison was made with children aged $<13$ years who required transfer for additional respiratory support for respiratory distress during the 12 months prior to the availability of HFNC oxygen therapy at the same institution (1 August 2014 31 July 2015).

Children commenced on HFNC oxygen according to departmental guidelines were identified from the ethics-approved NSH High-Flow Nasal Cannula Registry (University of Cape Town Human Research Ethics Committee (UCT HREC) ref. no. R051/2015). Children transferred to L3 care for respiratory support during the preceding year were identified from existing routine morbidity and mortality records of the Department of Paediatrics at NSH. The UCT HREC granted ethical approval for this study and waived the need for informed consent owing to its retrospective nature (ref. no. 534/2016), and the NSH CEO gave permission to use patient data.

\section{Setting}

NSH is a 330-bed L2 hospital, i.e. there are specialists on site, but no subspecialty or intensive care service is offered. The paediatric population served comprises $~ 100000$ children, mostly from middle and lower socioeconomic backgrounds and many from informal housing areas. There is no PICU on site, and children requiring advanced respiratory support need to be transported to an L3 hospital where subspecialty services and PICU facilities are available. The L3 hospital is 30 minutes away by road ambulance transport. Staffing (both nurse and doctor numbers) remained unchanged during the two study periods. All children with respiratory distress requiring oxygen therapy are closely monitored in the paediatric ward and receive supportive care, including nasogastric tube feeding if in moderate or severe respiratory distress.

\section{HFNC system and protocol}

The Fisher and Paykel Airvo 2 system (Fisher \& Paykel Healthcare Ltd, New Zealand) is used at NSH, with a humidifier and age- and weight-appropriate Fisher and Paykel nasal cannulas. The NSH HFNC oxygen guideline was adapted for local use from the Royal Children's Hospital, Melbourne, guideline. ${ }^{[14]}$ Starting flow rates are based on the weight of the child $(<10 \mathrm{~kg}: 2.0 \mathrm{~L} / \mathrm{kg} / \mathrm{min}$ and $>10 \mathrm{~kg}$ : $2.0 \mathrm{~L} / \mathrm{kg} / \mathrm{min}$ for the first $10 \mathrm{~kg}$ plus $0.5 \mathrm{~L} / \mathrm{kg} / \mathrm{min}$ for each $\mathrm{kg}$ above $10 \mathrm{~kg}$, with a maximum flow rate of $50 \mathrm{~L} / \mathrm{min}$ ). The decision to commence HFNC always involves a senior paediatrician. Both nurses and doctors working in the ward received basic training in the use of the device and the children receiving HFNC had hourly observations performed, including pulse oximetry and measurement of the respiratory rate (RR) and heart rate (HR), in a high-care bed.

The indications for HFNC in the guideline include use in children with moderate-to-severe respiratory distress with hypoxaemia (peripheral oxygen saturation $<90 \%$ ) despite standard low-flow oxygen (provided by nasal prong or face-mask) due to common childhood respiratory illnesses such as community-acquired pneumonia, bronchiolitis, and asthma exacerbations. Absolute contraindications to HFNC use include the need for immediate invasive respiratory support, infants or children with non-patent nasal passages, and infants weighing $<3 \mathrm{~kg}$.

Children receiving HFNC were monitored and reviewed both 30 minutes and $\sim 2$ hours after being initiated on HFNC to determine whether transfer to $\mathrm{L} 3$ and/or additional support was required. No improvement in the RR and HR ( $>20 \%$ reduction not achieved), inability to wean the inspired oxygen concentration to $<40 \%$, or signs of worsening respiratory distress indicated treatment failure and the need to transfer the child for advanced respiratory support.

\section{Data collection and definitions}

The medical folders of identified patients were drawn from the medical records department and reviewed, and relevant data were entered into an electronic data capture sheet (Microsoft Access version 2013; Microsoft, USA). Any results and details not recorded in the medical folder were searched for through the electronic National Health Laboratory Service and Clinicom platforms.

The data collected comprised basic demographic information, diagnosis, underlying comorbidity, birth weight, gestational age, and HIV and nutritional status. Diagnosis was as assigned by the attending ward doctor according to standard definitions of pneumonia (cough with fast breathing and/or chest in-drawing) or bronchiolitis (associated preceding coryzal illness with predominant features of hyperinflation with crackles and/or wheezing). HIV status was classified as uninfected, exposed but uninfected, and infected. Owing to the small number of HIV-infected children, no distinction was made according to whether these children were taking combined antiretroviral treatment (cART), were precART or had defaulted CART, or according to their immunological or virological status. The nutritional status of each child was determined by the World Health Organization (WHO) weight-forage (WAZ) and weight-for-height/length (WHZ) $z$-scores. These $z$-scores were calculated using the 'zanthro' function of Stata/IC 13.0 statistical software (StataCorp, USA), with 2007 UK WHO term and preterm growth charts used for reference (birth: British 1990 Growth Reference, reanalysed 2009; postnatal: WHO Child Growth Standards; 4 - 20 years: British 1990 Growth Reference - information 
accessible to registered Stata users). Prematurity was taken into account in the calculation of the $z$-scores. Malnutrition was defined as a WHZ score $<-2$ (moderate wasting). The method of delivery of standard low-flow oxygen support received by transferred children in the pre-intervention group was collected.

We obtained the baseline number of monthly admissions from routine departmental data on total admissions for acute LRTIs in children aged $<5$ years. Despite being restricted to children aged $<5$, we thought that this would be a reasonable approximation of the total number of acute LRTI admissions given that $90 \%$ of our total cohort was $<5$ years of age, and the older group were mostly children with asthma who would not be counted in the baseline data.

\section{Data analysis}

Data analysis was performed using Stata/IC 13.0 statistical software. Continuous data tested for normality using the Shapiro-Wilks test are presented as medians (interquartile range (IQR)) or means (standard deviation (SD)) for non-normally and normally distributed data, respectively. Medians and means were compared between groups using the Wilcoxon rank-sum or Student's $t$-test, respectively. Categorical data are presented as frequencies and percentages, with the $\chi^{2}$ or Fisher's exact test used for comparison between groups, as appropriate. For all tests, $p<0.05$ was considered significant.

\section{Outcome measures}

Outcomes described in the HFNC group included the length of time receiving HFNC, any severe adverse events associated with HFNC use, and success or failure of the intervention. Success was defined as no need for escalation of respiratory support, while failure was defined as needing further non-invasive (CPAP) or invasive (IPPV) respiratory support within 48 hours of transfer, or death during admission. The intervention's success and failure outcomes were then compared with similar outcomes in the pre-intervention (pre-HFNC) group.

\section{Results}

During the first 12 months of on-site availability of HFNC in the general paediatric ward (1 August 2015 - 31 July 2016), 66 infants and children received HFNC oxygen therapy. In the pre-HFNCavailability period (1 August 2014 - 31 July 2015), during which only standard low-flow oxygen respiratory support (nasal prong oxygen or face-mask oxygen) was available, 54 children required transfer to an L3 hospital for further respiratory support.

\section{Demographic and clinical characteristics}

The median age of the whole study cohort was 5 months (IQR 1.9 14.6), with a slight male preponderance (Table 1). Of the study

\section{Table 1. Demographic and clinical characteristics}

\begin{tabular}{|c|c|c|c|c|}
\hline & Total $(N=120)$ & $\begin{array}{l}\text { Pre-HFNC-availability } \\
\text { group }(N=54)\end{array}$ & $\begin{array}{l}\text { HFNC-availability } \\
\text { group }(N=66)\end{array}$ & $p$-value \\
\hline Age (months), median (IQR) & $5.0(1.9-14.6)$ & $3.2(1.4-13.2)$ & $6.4(2.5-14.9)$ & 0.057 \\
\hline Age $<5$ years, $n(\%)$ & $107(89.2)$ & $47(87.0)$ & $60(90.9)$ & 0.49 \\
\hline Sex, $n(\%)$ & & & & 0.60 \\
\hline Male & $68(56.7)$ & $32(59.3)$ & $36(54.6)$ & \\
\hline Female & $52(43.4)$ & $22(40.7)$ & $30(45.4)$ & \\
\hline \multicolumn{5}{|l|}{ Nutritional status } \\
\hline WAZ, mean (SD) & $-0.98(1.96)$ & $-1.38(1.90)$ & $-0.65(1.97)$ & 0.04 \\
\hline $\mathrm{WAZ}<-2, n(\%)$ & $37(30.8)$ & $23(42.6)$ & $14(21.2)$ & 0.01 \\
\hline HAZ, median (IQR) & $-0.53(-1.57-0.28)$ & $-0.53(-1.56--0.02)$ & $-0.48(-1.57-0.57)$ & 0.69 \\
\hline $\mathrm{HAZ}<-2, n(\%)$ & $22 / 114(19.3)$ & 9/48 (18.8) & $13 / 66(19.7)$ & 0.90 \\
\hline WHZ, median (IQR) & $-0.60(-1.38-0.86)$ & $-1.17(-2.09-0.60)$ & $-0.23(-0.88-0.92)$ & 0.002 \\
\hline WHA $<-2, n(\%)$ & $16 / 106(15.1)$ & $14 / 45(31.1)$ & $3 / 61(3.3)$ & $<0.001$ \\
\hline HIV status, $n(\%)$ & & & & $0.02^{*}$ \\
\hline Exposed & $23(19.2)$ & $13(24.1)$ & $10(15.2)$ & \\
\hline Infected & $10(8.3)$ & $8(14.8)$ & $2(3.0)$ & \\
\hline Uninfected & $87(72.5)$ & $33(61.1)$ & $54(81.8)$ & \\
\hline Comorbidities, $n(\%)$ & & & & $0.14^{*}$ \\
\hline Anaemia & $10 / 58(17.2)$ & $3 / 29(10.3)$ & $7 / 29(24.1)$ & \\
\hline Asthma & $14 / 58(24.1)$ & $5 / 29(17.2)$ & $9 / 29(31.0)$ & \\
\hline CLD & $4 / 58(6.9)$ & $3 / 29(10.3)$ & $1 / 29(3.5)$ & \\
\hline HIV-infected & $10 / 58(17.2)$ & $8 / 29(27.6)$ & $2 / 29(6.9)$ & \\
\hline Cardiac & $7 / 58(12.1)$ & $5 / 29(17.2)$ & $2 / 29(6.9)$ & \\
\hline Probable TB & $4 / 58(6.9)$ & $1 / 29(3.5)$ & $3 / 29(10.3)$ & \\
\hline Other & $9 / 58(15.5)$ & $4 / 29(13.8)$ & $5 / 29(17.2)$ & \\
\hline Prematurity, $n(\%)$ & $30(25.0)$ & $18(33.3)$ & $12(18.2)$ & 0.057 \\
\hline Diagnosis, $n(\%)$ & & & & $0.13^{*}$ \\
\hline Pneumonia & $84(70.0)$ & $42(77.8)$ & $42(63.6)$ & \\
\hline Bronchiolitis & $23(19.2)$ & $6(11.1)$ & $17(25.8)$ & \\
\hline Asthma & $13(10.8)$ & $6(11.1)$ & $7(10.6)$ & \\
\hline
\end{tabular}


population for whom nutritional data were available $(106 / 120), 15.1 \%(16 / 106)$ were moderately or severely malnourished, with a greater proportion in the pre-HFNC group $(31.1 \%, 14 / 45)$ than in the HFNC group $(3.3 \%, 3 / 61)$ being malnourished. A significantly greater proportion of children were HIV-infected or HIV-exposed in the pre-HFNC group than in the HFNC group. Among children with underlying comorbid conditions $(n=58)$, asthma and anaemia were the most common, being present in $24.1 \%(14 / 58)$ and $17.2 \%(10 / 58)$ of these children, respectively. In addition, $12.1 \%$ (7/58) had an underlying congenital cardiac abnormality. The most common diagnosis was pneumonia $(n=84,70.0 \%)$, followed by bronchiolitis, then asthma. Of children aged $<5$ years, the majority $(n=82,76.6 \%)$ had pneumonia, whereas in the older age group ( $>5$ years) the majority $(n=11,84.6 \%)$ had asthma. A quarter of the study population had been born prematurely.

\section{Comparison of outcomes between the groups}

\section{Death during admission}

Two children died in each group, 3.7\% in the HFNC-availability group and $3.0 \%$ in the pre-HFNC-availability group. The deaths in the HFNC-availability group were unrelated to the intervention. In this group, the children who died were 4 and 13 months old, HIV-unexposed and uninfected, not malnourished, and had an initial diagnosis of pneumonia. The 4-month-old child had no underlying comorbidities and, within 12 hours of commencing HFNC, was transferred to L3 for invasive respiratory support in the PICU; he died 8 weeks later of intractable respiratory failure secondary to severe bronchopneumonia. The 13-month-old child had significant underlying comorbidities Down syndrome, hypothyroidism, gastrooesophageal reflux disease and evidence of chronic lung disease. He received HFNC oxygen for 9 days during his stay and was weaned off successfully, but unexpectedly died of aspiration at the regional hospital after a 5-week hospital admission.

Need for escalation of respiratory support Fig. 1 shows the study flow. In the preHFNC-availability group, 54/835 children (6.5\%) admitted for acute LRTIs were assessed as needing transfer to $\mathrm{L} 3$ for possible additional respiratory support. Of the 54 children transferred, 38 (70.4\%) required CPAP or IPPV within 48 hours of transfer, or died, while $16(29.6 \%)$ received HFNC or remained on nasal prong oxygen at L3.

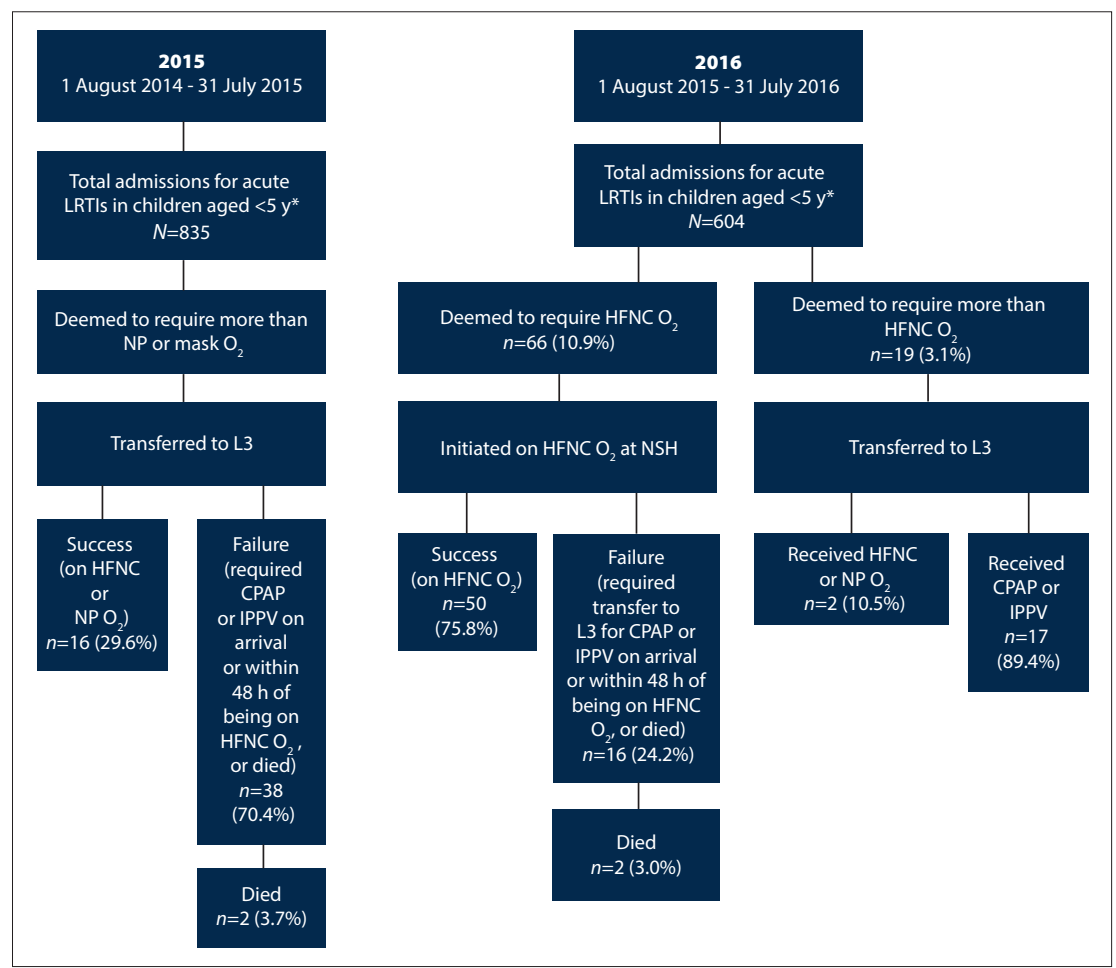

Fig. 1. Participant flow. $(y=$ years; $h=$ hours; LRTI = lower respiratory tract infection; NP = nasal prong; $\mathrm{O}_{2}=$ oxygen; $\mathrm{L} 3=$ level 3 hospital; $H F N C=$ high-flow nasal cannula; $C P A P=$ continuous positive airway pressure; IPPV = intermittent positive-pressure ventilation; NSH = New Somerset Hospital; ${ }^{*}$ Number of admissions for children aged $\geq 5$ y not available ( $89 \%$ of the overall cohort was $<5 y$ old).)

In the HFNC availability group, 85/604 (14\%) of children admitted for acute LRTIs were assessed as needing more than standard low-flow oxygen therapy; 66 (10.9\%) were initiated on HFNC in the general ward at NSH, while $19(3.1 \%)$ were assessed as needing more support than could be provided by HFNC and were transferred directly to L3. Overall, 33 children (5.5\%) admitted for acute LRTIs were transferred to L3. Of the 19 children assessed as requiring transfer to L3 without being offered on-site HFNC oxygen, the majority $(n=17,9.4 \%)$ received CPAP or IPPV at the referral facility, while $2(10.5 \%)$ were managed with HFNC or nasal prong oxygen. Of the 66 children initiated on HFNC in the general ward, $50(75.8 \%)$ were successfully managed and weaned off HFNC and discharged home. The other $16(24.2 \%)$ were assessed as not settling on HFNC and were transferred to L3 within 48 hours of being on HFNC. At L3 they received either CPAP or IPPV; one of these children died after a period of prolonged ventilation.

\section{The HFNC group}

\section{Length of time receiving HFNC}

The overall median length of time on HFNC was 46.3 hours (IQR 19.5 - 93.5). Of the 16 children who failed HFNC at
$\mathrm{NSH}$, the median length of time on HFNC was 12 hours (IQR 4 -28). Among those who were successfully managed on HFNC, the median length of time on HFNC was 58.5 hours (IQR 39.5 - 106). Four children required HFNC for longer than 7 days (range 9 days (231 hours) - 13 days (322 hours)); one of these patients died.

Serious adverse effects in the HFNC group No pneumothoraces, episodes of gastric or abdominal distension, mucosal injuries or infections were documented for any of the children receiving HFNC oxygen therapy in the general ward of NSH during the first 12 months of its use. Of the infants who failed HFNC at L2 and were transferred to L3, most were boys ( $n=12,75.0 \%)$ and the median age of the 16 infants was 6.7 months; $10(62.5 \%)$ were $<6$ months old. The attending doctor's interpretation of the chest radiographs was that 15 of the infants had hyperinflation and either focal areas of opacification or changes consistent with bronchopneumonia. At L3, 15 children had blood cultures performed (all results were negative) and $12(75.0 \%)$ had a nasopharyngeal aspirate (NPA) collected. Two children's NPA specimens were negative and $10(83.3 \%)$ tested positive for respiratory viruses: 5 were positive for adenovirus, 3 were positive for both adenovirus and respiratory 
syncytial virus (RSV), one specimen was only RSV-positive, and one was influenza virus-positive. The vital sign monitoring data (HR, RR, $\mathrm{FiO}_{2}$ and oxygen saturation) of the 16 children who failed HFNC, recorded hourly at L2, were reviewed, and no consistent pattern predicting failure was observed.

\section{Discussion}

This is the first study from sub-Saharan Africa documenting the safe, effective use of HFNC in a general paediatric ward setting. We identified no complications and demonstrated that an L2 hospital has the capacity to manage children who require more than standard lowflow oxygen respiratory support, reducing pressure on L3 services.

Pneumonia was the predominant diagnosis in the overall study population, reflecting current evidence that pneumonia is the leading cause of morbidity and mortality in children in SA. ${ }^{[2]}$ A significant proportion of the children in this study population had wasting (WHZ <-2) and $25.0 \%$ had been born prematurely. Asthma, anaemia and cardiac abnormalities were the most prevalent underlying comorbidities. Unlike the study by Chisti et al., ${ }^{[13]}$ in the present study an underlying cardiac abnormality was not a contraindication to the use of HFNC despite very limited evidence for its efficacy in paediatric cardiac patients. ${ }^{[15]}$ One child with a cardiac abnormality died, but not while receiving the intervention. Although the numbers were small, HFNC was successfully used in 4 children with underlying congenital heart disease as respiratory support during an acute admission for pneumonia.

The proportion of HIV-infected children was significantly lower in the HFNC availability group than in the pre-HFNC group; although it is unlikely, this may reflect the effectiveness of ongoing strategies to prevent perinatally acquired HIV infection in SA via prevention of mother-to-child transmission and the early initiation of cART.

HFNC use did not appear to affect the number of deaths in the two groups, a finding consistent with that reported by Chisti et al. ${ }^{[13]}$ Mortality rates were very low for both groups $(0.2$ and 0.3 deaths $/ 100$ admissions), so this study was underpowered to make any meaningful conclusions with regard to the effect of HFNC on mortality.

Transferring children from L2 to L3 hospitals puts a significant strain on limited resources, including the emergency medical services and L3 bed capacity. Whether a transfer is warranted and safe raises anxiety and concern among staff at the referring facility. A much higher proportion of children required additional respiratory support during the year when HFNC was available than during the preceding year $(14.1 \%$ v. $6.5 \%)$. It is possible that this was due to a 'very bad respiratory season' (anecdotal). It is unlikely that it was simply because of availability of HFNC, as the intervention had been available at the L3 facility in the preceding years, criteria regarding requirements for additional respiratory support were the same for both institutions, and children identified as needing the intervention would have been transferred. The transfers would not have been accepted by the L3 institute if deemed unnecessary, and all decisions were consultant led. Therefore, almost two-thirds of the children who would potentially have been transferred prior to the availability of HFNC at L2 were successfully managed at L2 in 2016, reducing staff anxiety about observing and transferring very ill children, and decreasing the strain on the emergency medical services and L3 bed capacity.

Although the total number of cases of severe acute asthma was small, children receiving HFNC as part of management responded well with no adverse events. It could be proposed that use of HFNC be included as an addition to current standard asthma management guidelines as an adjunct at the stage of using intravenous medication (salbutamol and magnesium sulphate) in severe acute exacerbations not responding to standard nebulised therapy.

A lower proportion overall of children transferred after receiving initial HFNC at L2 needed non-invasive or invasive respiratory support at L3 once transferred, compared with children transferred on/after receiving only standard LFNC oxygen. This supports the findings of Wing et al. ${ }^{[16]}$ that the introduction of HFNC reduced the need for intubation and the need for non-invasive/invasive retrieval during interhospital transfer. Because we used retrospective data from the pre-HFNC-availability cohort, we do not have information on how long children were at the referring facility prior to transfer and whether this could have affected outcome. Fewer children requiring high-care/PICU non-invasive or invasive ventilatory support at L3 also reduces the strain on limited L3 resources (high-care and PICU bed capacity and nursing requirements), justifying the provision of HFNC therapy at lower levels of care. In the HFNC-availability group, the majority of children assessed as needing more support than that provided by HFNC (i.e. those who did not meet the requirements of the protocol for safe use of HFNC at L2) ultimately required either CPAP or IPPV at L3. These findings support the current clinical practice and endorse the current protocol.

The NSH HFNC protocol states that if there is no objective response to HFNC (reduction in HR and RR within $2-4$ hours), the intervention has failed and the child needs transfer for additional support (CPAP or IPPV). The median length of time that HFNC oxygen was provided was 12 hours, which may indicate that this protocol was not strictly adhered to and that an earlier decision to transfer may have been preferable. It could also reflect natural disease progression with some patients deteriorating during the admission, requiring increased support because of hypoxia or tiring, or that some patients such as asthmatics needed shorter periods of support.

The absence of any recorded serious adverse events related to the use of HFNC in our general paediatric ward is encouraging and concurs with the literature reporting few adverse effects. ${ }^{[15,17,18]}$ Lack of adverse events further supports the use of HFNC as an attractive option, but strict adherence to protocol is required. Although they were reviewed, detailed analysis of the group of children who failed HFNC at L2 was not done because we did not have the corresponding data from children who did not fail for comparison. However, there appeared to be an association between the doctor's assessment of RR at initiation of HFNC and the documented RR at transfer, with patients who were transferred remaining significantly tachypnoeic. The high proportion of infants with severe pneumonia needing transfer to L3 for respiratory support who had adenovirus on NPA supports the recently published study by Zampoli and MukuddemSablay ${ }^{[19]}$ demonstrating that adenovirus is an important cause of severe pneumonia necessitating ICU admission in young children in SA.

\section{Study limitations}

This study has several limitations. Its design was retrospective, using an unmatched, historical comparator group. However, the criteria for additional respiratory support were the same and the staff was unchanged. Although the time period was similar (including one autumn/winter respiratory season in both groups) and the referral areas and admission criteria were the same, it seems that there were more admissions in the first group and that the severity of illness necessitating additional respiratory support was greater in the second group. Analysis of the group of children who failed HFNC at L2 was not included, as we did not have corresponding data from children who did not fail, for comparison. 


\section{Conclusions and recommendations}

HFNC is a safe, effective, feasible option for non-invasive respiratory support in a relatively resource-limited setting as an adjunct in the management of respiratory illnesses in children, with reduced work of breathing and respiratory rates in those children who were successfully managed in a general paediatric ward. The lack of serious adverse events is encouraging, but there is a need to refine recognition of early failures and to avoid delays in transfer. A detailed cost analysis was not done, but despite the relative cost of disposables and equipment, broader cost reductions are anticipated: decreased inter-hospital transfers, avoidance of L3 admissions, and reduced bed pressure in a setting known to have a shortage of PICU and high-care space. Results of ongoing international randomised clinical trials are eagerly anticipated to further guide appropriate use in similar settings.

Declaration. This research was performed as part of EH's MMed dissertation.

Acknowledgements. We would like to acknowledge Dr Mark Richards for establishing the High-Flow Nasal Cannula Registry at NSH (UCT HREC ref. no. R051/2015), which allowed identification of patients receiving the intervention. We would also like to acknowledge the NSH Facilities Board for donation of the equipment and consumables that allowed us to begin using this intervention in our ward.

Author contributions. EH performed the research as part of her MMed dissertation, so was involved in protocol design and submission, data collection, analysis and interpretation. KLR was involved with study and database design, data analysis, editing and referencing. MLC was MMed supervisor involved in protocol design and submission, and editing and revision of content for the dissertation and for journal submission.

Funding. Internal funding was received from a Department of Education grant to the University of Cape Town for MMed students to assist with data analysis only.

Conflicts of interest. None.
1. Williams BG, Gouws E, Boschi-Pinto C, Bryce J, Dye C. Estimates of world-wide distribution of child deaths from acute respiratory infections. Lancet Infect Dis 2002;2(1):25-32. https://doi.org/10.1016/ deaths from acute respir

2. World Health Organization. WHO Methods and Data Sources for Country-level Causes of Death 2000 World Health Organization. WHO Methods and Data Sources for Country-level Causes of Death 2000
- 2012. Global Health Estimates Technical Paper WHO/HIS/HS/GHE/20147. Geneva: Department of Health Statistics and Information Systems, WHO, 2014

3. Reid AE, Hendricks MK, Groenewald P, Bradshaw D. Where do children die and what are the causes? Under-5 deaths in the Metro West geographical service area of the Western Cape, South Africa, 2011. S Afr Med J 2016;106(4):359-364. https://doi.org/10.7196/SAMJ.2016.v106i4.10521

4. Duke T, Mgone J, Frank D. Hypoxaemia in children with severe pneumonia in Papua New Guinea. Int J Tuberc Lung Dis 2001;5(6):511-519. https://doi.org/10.1136/adc.86.2.108

5. Lozano J. Epidemiology of hypoxaemia in children with acute lower respiratory infection. Int J Tuberc Lung Dis 2001;5(6):496-504. https://doi.org/10.1007/BF02790384

6. Argent AC, Biban P. What's new on NIV in the PICU: Does everyone in respiratory failure require endotracheal intubation? Intensive Care Med 2014;40(6):880-884. https://doi.org/10.1007/s00134014-3274-z

7. Haq I, Gopalakaje S, Fenton AC, McKean MC, J O'Brien C, Brodlie M. The evidence for high flow nasal cannula devices in infants. Paediatr Respir Rev 2014;15(2):124-134. https://doi.org/10.1016/j. prrv.2013.12.002

8. Kubicka ZJ, Limauro J, Darnall RA. Heated, humidified high-flow nasal cannula therapy: Yet another way to deliver continuous positive airway pressure? Pediatrics 2008;121(1):82-88. https://doi. org/10.1542/peds.2007-095

9. Dysart K, Miller TL, Wolfson MR, Shaffer TH. Research in high flow therapy: Mechanisms of action. Respir Med 2009;103(10):1400-1405. https://doi.org/10.1016/j.rmed.2009.04.007

10. Holleman-Duray D, Kaupie D, Weiss MG. Heated humidified high-flow nasal cannula: Use and a neonatal early extubation protocol. J Perinatol 2007;27(12):776-781. https://doi.org/10.1038/ sj.jp.7211825

11. Morley C. Continuous distending pressure. Arch Dis Child Fetal Neonatal Ed 1999;81(2):F152-F156. https://doi.org/10.1136/fn.81.2.F152

12. Spence KL, Murphy D, Kilian C, McGonigle R, Kilani RA. High-flow nasal cannula as a device to provide continuous positive airway pressure in infants. J Perinatol 2007;27(12):772-775. https://doi. org/10.1038/s.jp. 7211828

13. Chisti MJ, Salam MA, Smith JH, et al. Bubble continuous positive airway pressure for children with severe pneumonia and hypoxaemia in Bangladesh: An open, randomised controlled trial. Lancet 2015;386(9998):1057-1065. https://doi.org/10.1016/S0140-6736(15)60249-5

14. Royal Children's Hospital, Melbourne, Australia. Clinical Practice Guideline on High Flow Nasal Prong (HFNP) therapy [last updated May 2014]. http://www.rch.org.au/clinicalguide/index.cfm (accessed 15 January 2018).

15. Testa G, Iodice F, Ricci Z, et al. Comparative evaluation of high-flow nasal cannula and conventional oxygen therapy in paediatric cardiac surgical patients: A randomized controlled trial. Interact Cardiovasc Thorac Surg 2014;19(3):456-461. https://doi.org/10.1093/icvts/ivu171

16. Wing R, James C, Maranda LS, Armsby CC. Use of high-flow nasal cannula support in the emergency department reduces the need for intubation in pediatric acute respiratory insufficiency. Pediatr Emerg Care 2012;28(11):1117-1123. https://doi.org/10.1097/PEC.0b013e31827122a9.

17. Hegde S, Prodhan P. Serious air leak syndrome complicating high-flow nasal cannula therapy: A report of 3 cases. Pediatrics 2013;131(3):e939-e944. https://doi.org/10.1542/peds.2011-3767

18. Jhung MA, Sunenshine RH, Noble-Wang J, et al. A national outbreak of Ralstonia mannitolilytica associated with use of a contaminated oxygen-delivery device among pediatric patients. Pediatrics associated with use of a contaminated oxygen-delivery device

19. Zampoli M, Mukuddem-Sablay Z. Adenovirus-associated pneumonia in South African children: . Zampoli M, Mukuddem-Sablay Z. Adenovirus-associated pneumonia in South African children:
Presentation, clinical course and outcome. S Afr Med J 2017;107(2):123-126. https://doi.org/10.7196/ SAMJ.2017.v107i2.11451

Accepted 6 September 2018. 